\title{
Intracrine VEGF signalling mediates colorectal cancer cell migration and invasion
}

\author{
Rajat Bhattacharya ${ }^{\star}{ }^{1}$, Fan Fan ${ }^{1}$, Rui Wang ${ }^{1}$, Xiangcang Ye ${ }^{1}$, Ling Xia ${ }^{1}$, Delphine Boulbes ${ }^{1}$ and Lee M Ellis ${ }^{\star}, 1,2$ \\ ${ }^{1}$ Department of Surgical Oncology, The University of Texas MD Anderson Cancer Center, Houston, TX 77030, USA and \\ ${ }^{2}$ Department of Molecular and Cellular Oncology, The University of Texas MD Anderson Cancer Center, Houston, TX 77030, USA
}

Background: Vascular endothelial growth factor (VEGF) and its receptors (VEGFRs) are key regulators of angiogenesis, affecting endothelial cell survival and function. However, the effect of VEGF-VEGFR signalling on tumour cell function is not well understood. Our previous studies in colorectal cancer (CRC) cells have demonstrated an intracrine VEGF/VEGFR1 signalling mechanism that mediates CRC cell survival and chemo-sensitivity. Since extracellular VEGF signalling regulates migration of endothelial cells and various tumour cells, we attempted to determine whether intracrine VEGF signalling affects CRC cell motility.

Methods: Migration and invasion of CRC cells, with and without VEGF or VEGFR1 depletion, were assayed using transwell migration chambers. Changes in cell morphology, epithelial-mesenchymal transition (EMT) markers, and markers of cell motility were assessed by immunostaining and western blot.

Results: Depletion of intracellular VEGF and VEGFR1 in multiple CRC cell lines led to strong inhibition of migration and invasion of CRC cells. Except for Twist, there were no significant differences in markers of EMT between control and VEGF/NEGFR1depleted CRC cells. However, VEGF/VEGFR1-depleted CRC cells demonstrated a significant reduction in levels of phosphorylated focal adhesion kinase and its upstream regulators pcMET and pEGFR.

Conclusions: Inhibition of intracrine VEGF signalling strongly inhibits CRC cell migration and invasion by regulating proteins involved in cell motility.

Vascular endothelial growth factor (VEGF) is a key regulator of angiogenesis and vascular function. Vascular endothelial growth factor promotes angiogenesis in numerous pathologic conditions, including cancer and ocular diseases (Connolly et al, 1989; Leung et al, 1989; Plouet et al, 1989). Its importance in development and physiology is demonstrated by embryonic lethality in mice with deletion of a single VEGF allele (Ferrara et al, 1996; Gerber et al, 1999). Vascular endothelial growth factor is also the most prominent growth factor to mediate endothelial cell proliferation and survival (Gerber et al, 1998a,b). These effects are attributed to well-studied canonical paracrine and autocrine VEGF signalling effects on endothelial cells (Lee et al, 2007a). The mechanisms of VEGF signalling in angiogenesis have been extensively studied (Ferrara et al, 2003).

Although the effects of VEGF and its receptors have been well characterised in endothelial cells, the role of VEGF in tumour cell function remains poorly understood. Lee et al (2007b) showed that an intracrine pathway might mediate cell survival in breast cancer cells. Work from our laboratory has demonstrated that VEGF plays an important role in tumour cell survival and modulates the sensitivity of tumour cells to chemotherapy (Samuel et al, 2011). Our studies utilising depletion of VEGF by somatic knockout of the VEGF gene (Samuel et al, 2011) or by siRNA knockdown in human colorectal cancer (CRC) cell lines (Bhattacharya et al, 2016) have demonstrated that loss of VEGF expression significantly decreased cell proliferation, increased apoptosis, and enhanced chemo-sensitivity of CRC cells (Samuel et al, 2011; Bhattacharya et al, 2016). Importantly, these effects were not mediated in an autocrine or paracrine fashion, as neutralisation of extracellular VEGF with a monoclonal antibody or inhibition of VEGF receptor (VEGFR) kinase activity did not alter cell survival. These findings

*Correspondence: Dr R Bhattacharya; E-mail: rbhattacharya@mdanderson.org or Professor LM Ellis; E-mail: lellis@mdanderson.org

Received 11 April 2017; revised 19 June 2017; accepted 30 June 2017; published online 25 July 2017

(C) 2017 Cancer Research UK. All rights reserved 0007 - 0920/17 
suggest that the intracellular effects of VEGF were independent of canonical VEGF-VEGFR activity (Samuel et al, 2011; Bhattacharya et al, 2016). Cumulatively, these studies demonstrated that VEGF functions in CRC cells via a novel intracrine mechanism that is distinct from the established paracrine or autocrine roles of VEGF in angiogenesis. These intracrine effects were mediated through VEGF by regulation of the activity of multiple receptor tyrosine kinases (RTKs; e.g., EGFR and cMET) and downstream AKT signalling (Bhattacharya et al, 2016) in CRC cells. Similar effects in these cells were observed upon depletion of VEGFR1, supporting the role of a novel intracellular VEGF-VEGFR1 complex in CRC cell survival (Bhattacharya et al, 2016).

Apart from its pro-survival effects, VEGF signalling also mediates migration in numerous cell types. Previous studies have shown that VEGF signalling induces migration of endothelial cells in normal physiologic processes and in tumours (Koch and Claesson-Welsh, 2012). Inhibition of paracrine VEGF signalling has been shown to inhibit endothelial cell motility (Podar et al, 2006; Costa et al, 2009). Our studies and studies from other groups have also shown that paracrine/autocrine VEGF signalling, through its receptors, mediates migration of various tumour cells (Fan et al, 2005; Lu et al, 2012; Sennino et al, 2012). Since VEGF signalling has been shown to mediate migration of endothelial cells and various tumour cells and since our prior studies demonstrated the presence of an intracrine signalling pathway in CRC cells, we sought to determine whether inhibition of intracrine VEGF signalling affects CRC cell motility. In this study, we show that inhibition of intracrine VEGF signalling strongly reduces CRC cell migration and invasion. Our study also indicates that these changes in migration are not due to epithelial-mesenchymal transition (EMT)-like changes in CRC cells but, rather, are due to a decrease in the activity of proteins associated with cell motility.

\section{MATERIALS AND METHODS}

Cell culture. The human CRC cell lines HCT116, SW480, SW620, and HT29 were purchased from American Type Culture Collection (ATCC; Manassas, VA, USA). The CRC cells were cultured in minimum essential medium (MEM) supplemented with $10 \%$ foetal bovine serum (FBS; Atlanta Biologicals, Flowery Branch, GA, USA) and the recommended concentrations of vitamins, nonessential amino acids, penicillin/streptomycin, sodium pyruvate, and L-glutamine (Thermo Fisher Scientific, Waltham, MA, USA). All experiments were performed using cells within 10-12 passages. The plasmid expressing FLAG-cMET was a generous gift from Dr Mien-Chie Hung (Du et al, 2016) at The University of Texas MD Anderson Cancer Center, and the plasmid expressing green fluorescent protein (GFP)-focal adhesion kinase (FAK; Gu et al, 1999) was obtained from Addgene (Cambridge, MA, USA).

Reagents. Recombinant human VEGF-A was purchased from R\&D Systems, Inc. (Minneapolis, MN, USA) and used at $20 \mathrm{ng} \mathrm{ml}^{-1}$. Bevacizumab was obtained from the pharmacy at MD Anderson Cancer Center. Human immunoglobulin G (IgG) was purchased from Innovative Research, Inc. (Novi, MI, USA). Pazopanib and axitinib (VEGFR1 inhibitors) and defactinib (a FAK inhibitor) were purchased from Selleck Chemicals (Houston, TX, USA).

Western blotting. Proteins in cell lysates or concentrated supernatant media $(\mathrm{CM})$ (concentrated by Ultracel-3K centrifugal filter units; EMD Millipore, Billerica, MA, USA) were separated by sodium dodecyl sulphate polyacrylamide gel electrophoresis following standard protocol and transferred to Immobilon polyvinylidene membranes (EMD Millipore). Membranes were blocked with 5\% milk in Tris-buffered saline with $0.1 \%$ Tween 20 (TBST) for $1 \mathrm{~h}$ followed by incubation with primary antibody (diluted in blocking buffer or $2 \%$ bovine serum albumin in TBST) overnight. Membranes were then washed three times in TBST and re-incubated with horseradish peroxidase-labelled secondary antibodies for $1 \mathrm{~h}$, washed three times in TBST, and exposed to autoradiography films. Signals were detected by chemiluminescence (Thermo Fisher Scientific). Antibodies for N-cadherin, E-cadherin, pcMET Y1234/Y1235, pEGFR Y1068, pFAK Y925, pSRC Y416, vimentin, Snail, and Twist were from Cell Signaling Technology (Danvers, MA, USA). The antibody against pVEGFR1 (Y1213) was purchased from R\&D Systems Inc.. Antibodies for VEGF, vinculin, $\alpha$-tubulin, Zeb1, and actin were from Santa Cruz Biotechnology (Dallas, TX, USA). All antibodies were used according to manufacturers' specifications. All cell lysates were prepared in radioimmunoprecipitation assay buffer with protease and phosphatase inhibitors.

siRNA knockdown. A mixture of three different siRNAs targeting all isoforms of human VEGF were used to deplete VEGF in CRC cells (Bhattacharya et al, 2016). Similarly, VEGFR1 was depleted using a mixture of two different siRNAs targeting the N-terminus of VEGFR1 as previously described (Bhattacharya et al, 2016). A validated non-targeting siRNA obtained from Sigma-Aldrich (St. Louis, MO, USA) was used as a control. Lipofectamine 2000 siRNA transfection reagent was used according to the manufacturer's instructions (Thermo Fisher Scientific). The siRNA knockdown experiments were performed in six-well dishes in regular cell culture medium containing 5\% FBS and without antibiotics. Twenty-four hours after transfections, cells were washed with phosphate-buffered saline (PBS) and cultured with MEM supplemented with $1 \%$ FBS and antibiotics. Forty-eight hours later, supernatant media were collected and assayed for VEGF depletion, or cells were lysed and assayed for various proteins.

For cMET depletion, siRNAs targeting cMET (Silencer Select siRNA \#4390824; Thermo Fisher Scientific) were used according to the manufacturer's protocol.

Migration and invasion assays. Cell migration in response to increased serum concentrations was assessed using modified Boyden chambers according to the manufacturer's protocol (Corning, Bedford, MA, USA). In brief, CRC cells were transfected with control-siRNA, VEGF- siRNA, or VEGFR1-siRNA. Approximately $48 \mathrm{~h}$ after transfection, HCT116 (50 000 cells), SW480 cells (100 000 cells), SW620 (100000 cells), or HT29 (100 000 cells) in $1 \%$ MEM-FBS were incubated on membrane inserts with $8.0-\mu \mathrm{m}$ pores in 24 -well plates. Chemo-attractants (10\% MEM-FBS) were placed in the bottom wells. After $24 \mathrm{~h}$ (HCT116) or $48 \mathrm{~h}$ (other cell lines), cells that did not migrate were removed from the top side of the inserts with a cotton swab. Cells that had migrated to the underside of the inserts were stained with Diff-Quik (Harleco, Gibbstown, NJ, USA) and counted from at least three random fields at $\times 200$ magnification.

For invasion assays, similar methods were used, except that the cells were placed in the top compartment of modified Boyden chambers with Matrigel-coated membrane inserts (Corning). The numbers of invading cells were quantified from five random fields at $\times 200$ magnification.

Immunofluorescent staining. CRC cells were grown on round coverslips and fixed with $2 \%$ paraformaldehyde in PBS. Next, slides were treated with PBS containing 1\% Tween 20 to permeabilise the cells and washed three times with TBST. Slides were then blocked in 8\% fish gelatin in TBST (blocking buffer). After three washes in TBST, slides were incubated with primary antibodies diluted in blocking buffer for $1 \mathrm{~h}$ at room temperature. Slides were then washed again as above and incubated with secondary antibodies diluted in blocking buffer for $1 \mathrm{~h}$ at room temperature. Stained cells were then visualised using a confocal microscope with $\times 40$ or $\times 60$ oil objectives, and images were obtained with a 
charge-coupled device camera. DNA was visualised by staining with 4',6-diamidino-2-phenylindole. Antibodies targeting E-cadherin (BD Biosciences, San Jose, CA, USA), $\beta$-catenin (EMD Millipore), and ZO-1 (Zymed Laboratories, South San Francisco, CA, USA) were used at 1:200 dilution in blocking buffer.

Phosphatase treatments. HCT116 cells were transfected with VEGF-targeting siRNAs, and $24 \mathrm{~h}$ after transfection, the medium was replaced by MEM with $1 \%$ FBS and antibiotics, and the cells were further incubated for $24 \mathrm{~h}$. Next, the cells were treated with sterile $100 \mu \mathrm{M} \quad \mathrm{Na}_{3} \mathrm{VO}_{4}$ (sodium orthovanadate) for $\sim 8 \mathrm{~h}$, trypsinized, and re-plated on membrane inserts with $8.0-\mu \mathrm{m}$ pores for migration assays in the presence of sterile $100 \mu \mathrm{M} \mathrm{Na} \mathrm{NO}_{4}$. Control-siRNA-transfected cells were treated similarly. Untreated CRC cells transfected with control-siRNA or VEGF-siRNA were assayed as controls.

Statistical analyses. All graphical calculations and plotting of numerical data were performed using Excel (Microsoft, Redmond, WA, USA). All data are expressed as mean \pm standard error. The statistical significance of differences between experimental groups was determined by Student's $t$-test, and $P$-values $<0.05$ were considered significant.

\section{RESULTS}

Depleting VEGF led to reduced migration in CRC cells. In our previous studies, we determined that inhibition of intracrine VEGF signalling reduced CRC cell survival (Samuel et al, 2011; Bhattacharya et al, 2016). These effects were due to VEGFregulated phosphatase or phosphatases that reduced survival signalling in CRC cells. To determine whether VEGF depletion also affects cellular processes that are involved in tumour metastasis, we measured the effects of VEGF depletion on migration and invasion of tumour cells. Four CRC cell lines with different genetic backgrounds - HCT116, SW480, SW620, and HT29 - were transiently transfected with VEGF-targeting siRNAs (VEGF-siRNAs), and migration was determined using in vitro transwell migration assays with high (10\%) FBS-containing media as the chemo-attractant. CRC cells transfected with a non-targeting siRNA were used as controls. Also, control-siRNA-transfected CRC cells were further treated with bevacizumab or human IgG (control) to rule out the involvement of paracrine or autocrine VEGF signalling effects on CRC cell migration. The depletion of VEGF was verified by measuring secreted VEGF in supernatant media of transiently transfected CRC cells (Figure 1A). All cell types demonstrated a significant reduction in cell migration upon intracellular VEGF depletion with siRNA (Figure 1B). However, inhibiting extracellular VEGF with high doses of bevacizumab failed to alter cell migration (Figure 1B).

Our previous studies have shown that VEGF-siRNA transfection leads to a $\sim 30 \%$ increase in HCT116 cell apoptosis (Bhattacharya et al, 2016), while control-siRNA transfection does not affect HCT116 cell survival. Hence, VEGF-siRNA-treated HCT116 cells were plated at twice the number (100 000 cells) than control-siRNA-treated HCT116 cells (50000 cells), to compensate for possible loss in number of migrating cells due to cell death during the assay. Even this doubling of initial cell number failed to increase the migration rates of VEGF-depleted cells (Supplementary Figure 2), indicating that cell survival was not the determining factor in the altered migration of the VEGFdepleted cells.

To demonstrate that VEGF depletion also affects migration in other tumour cell types, we performed limited studies with MKN74 (gastric cancer cell line), SKOV3IP and HeyA8 (ovarian cancer cell lines) and MDA MB231 (breast cancer cell line). Previously published literature and unpublished studies indicated a possible intracrine VEGF signalling axis in these cell types. Depletion of VEGF resulted in significant reduction in migration in these cell lines (Supplementary Figure 1).

Invasion is significantly reduced in VEGF-depleted CRC cells. Next, we used HCT116 and SW480 cells to determine the effects of VEGF depletion on invasion in response to stimuli (high serum). Again, HCT116 and SW480 cells transfected with VEGF-siRNA invaded the membranes at significantly lower rates than did cells treated with control-siRNA (Figure 1C). Bevacizumab treatment had no effect on cell invasion (Figure 1C). We have not shown results for HT29 and SW620 cell invasion, as those cells failed to invade through the Matrigel-coated membranes within our experimental time period of $48 \mathrm{~h}$ and thus could not be used for the study.

VEGF depletion does not affect EMT molecular alterations in CRC cells. Tumour cell motility and invasion can be altered by changes in properties that dictate EMT (Kalluri and Weinberg, 2009; Scheel and Weinberg, 2012). To determine whether intracellular VEGF signalling regulated EMT in CRC cells, we looked for changes in the known molecular markers of EMT. Initial morphologic examination of CRC cells treated with either control-siRNA or VEGF-siRNA revealed no differences in the shape or size of HCT116 or SW480 cells (Figure 2A). Cells treated with bevacizumab or human IgG had no significant changes either. Similar results were obtained with SW620 and HT29 cells (data not shown). Western blots done on cell lysates failed to show significant differences due to VEGF depletion in most of the molecular markers (E-cadherin and $\mathrm{N}$-cadherin) associated with EMT in CRC cells (Thiery and Sleeman, 2006; Yang and Weinberg, 2008) that were assessed (Figure 2B). The transcription factors Snail, Twist, and Zeb1 have previously been shown to play an important role in mediating the changes observed with EMT (Cano et al, 2000; Yang et al, 2004; Eger et al, 2005). Most of these transcriptional factors were unaffected by the presence or absence of intracellular VEGF. Only Twist was found to be reduced in CRC cells depleted of VEGF (Figure 2B). Together, these studies indicate that the significant reduction in cell migration and invasion in VEGF-depleted CRC cells is not due to EMT alteration. Similar observations were made with SW620 and HT29 cells (Supplementary Figure 3).

Breakdown of cell-cell adhesion is one of the hallmarks of EMT (Kalluri and Weinberg, 2009). Disruption of the cell adhesion complex leads to alterations in the localisation of $\beta$-catenin and E-cadherin from their usual membrane-bound positions. Previous studies have shown that migration of $\beta$-catenin to the nucleus is associated with altered cell migration (Muller et al, 2002; Scheel et al, 2011). However, immunostaining of CRC cells with antibodies against $\beta$-catenin (Supplementary Figure 4A) failed to show any differences between control-siRNA-treated and VEGFsiRNA-treated cells in $\beta$-catenin localisation. Similarly, no significant changes in E-cadherin localisation were observed in response to VEGF depletion in CRC cells (Supplementary Figure 4A). We also examined $\mathrm{ZO}-1$, a protein involved in cell adherence (Anderson et al, 1995; Supplementary Figure 4B). Immunostaining of these markers did not indicate any changes or losses in expression or localisation of these proteins in VEGFdepleted CRC cells compared with control cells.

Motility-associated proteins are negatively affected by absence of intracellular VEGF in CRC cells. Cell motility is a complex process requiring interplay of multiple proteins, including cytoskeletal and cell membrane-associated proteins, specifically proteins at focal adhesions. As our studies failed to indicate changes in EMT, we investigated the effects of VEGF depletion on proteins that are known to affect the function of focal adhesions and cell motility. FAK has been extensively studied for its role in 
A

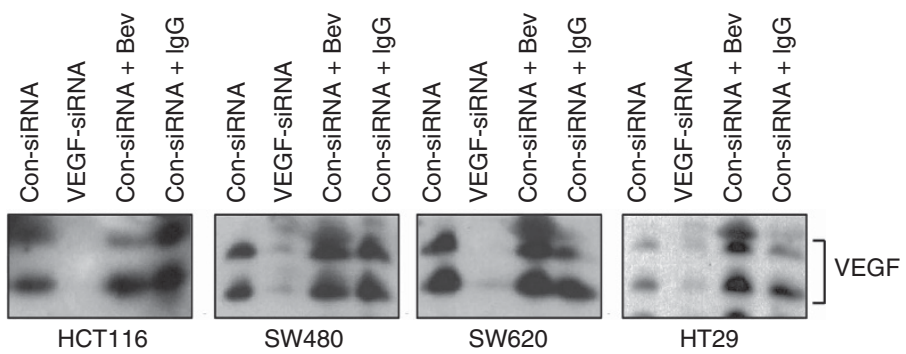

B

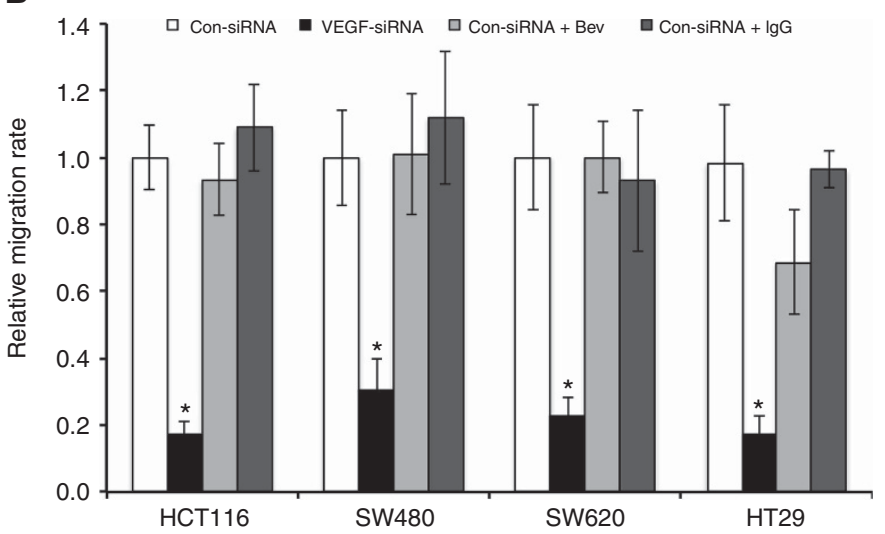

C
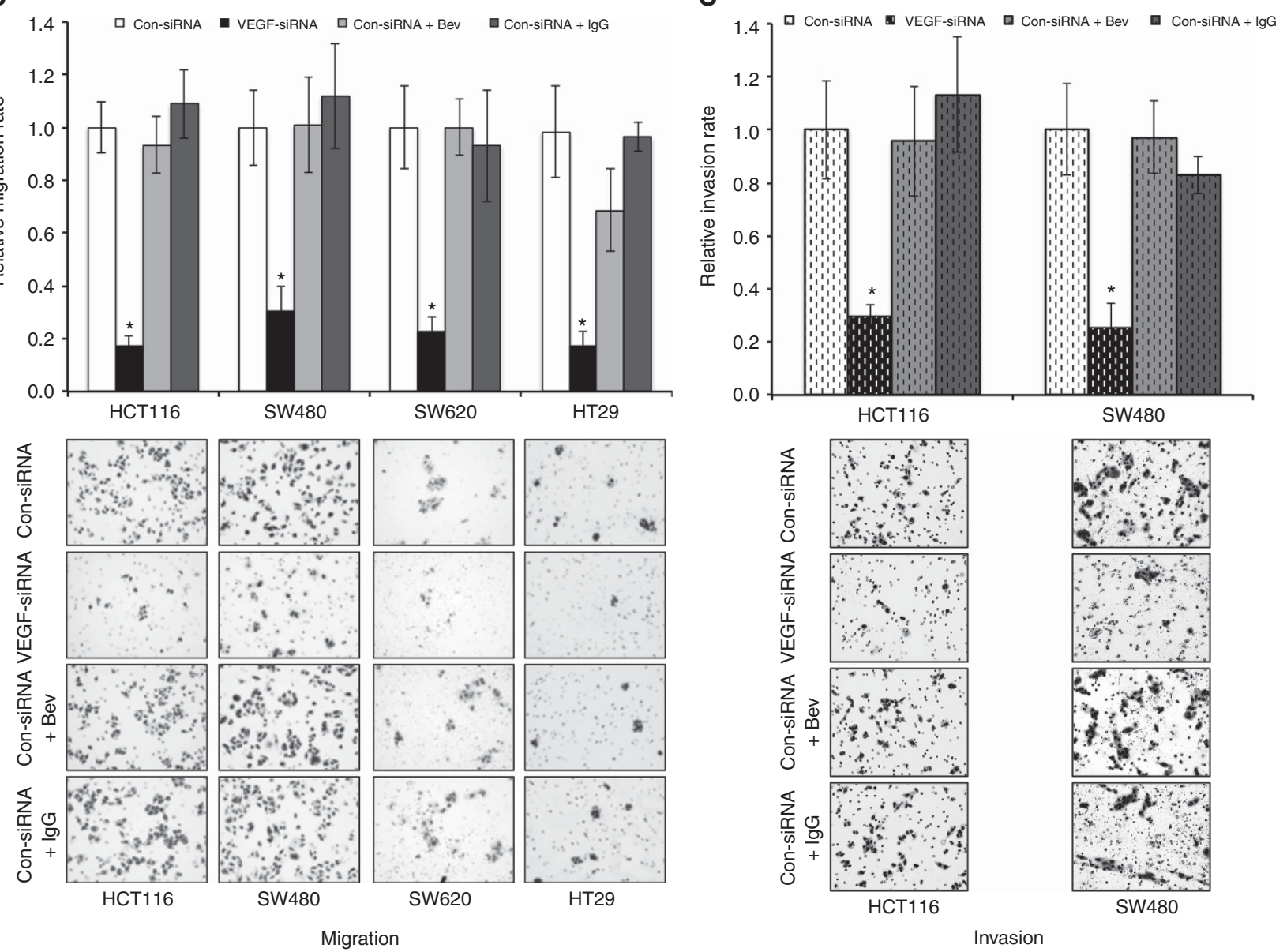

Figure 1. Depletion of intracellular VEGF inhibits CRC cell migration and invasion. (A) HCT116, SW480, SW620, and HT29 cells were transfected with siRNAs targeting all human VEGF isoforms and assayed for changes in VEGF expression. Western blots showing multiple isoforms of secreted VEGF in supernatant media collected from control-siRNA (Con-siRNA)-treated and VEGF-siRNA-treated cells validate that VEGF-siRNAs effectively depleted VEGF in these cell lines. Also shown are control cells treated with Con-siRNA and either bevacizumab (Con-siRNA + Bev) or human IgG (Con-siRNA + IgG). (B) HCT116, SW480, SW620, and HT29 cells were assayed for migration using transwell migration chambers. A significant decrease in migration was observed in CRC cells depleted of VEGF (using VEGF-siRNA) but not in cells with normal VEGF levels. The plots show relative migration rates derived from multiple experiments. The images below the plots show migrated cells and are representative of each experimental set used to calculate cell migration rate. ${ }^{*} P<0.005$. (C) Invasion of HCT116 and SW480 cells were measured using transwell migration chambers with Matrigel-coated membranes. The plots show relative invasion rates determined from multiple experiments, and the images below are representative images of cells that invaded through the coated membranes and that were used to calculate invasion rates. $\star P<0.005$.

cell migration and angiogenesis (Mitra and Schlaepfer, 2006; Zhao and Guan, 2011). Previous studies have indicated that phosphorylation of Y925 of FAK is important in regulating cell migration and angiogenesis (Mitra et al, 2006; Deramaudt et al, 2011). This activated form of FAK has also been shown to be important in VEGF-VEGFR-mediated migration of tumour cells (Lu et al, 2012). Our Western blot experiments to probe for pFAK Y 925 clearly indicated a strong reduction in the levels of pFAK (Figure 3) in cell lysates from CRC cells treated with VEGF-siRNA compared with the same cells treated with control-siRNA. There are multiple proteins upstream of FAK whose activities have strong effects on cell motility, including multiple RTKs (Mitra et al, 2005; Mitra and Schlaepfer, 2006). CRC cells express various RTKs, and we analysed pEGFR and pcMET in HCT116 cells and pEGFR and pSRC in SW480 cells, proteins that are reported to affect FAK function (Mitra et al, 2005). Robust decreases in pEGFR and pcMET were observed in VEGF-depleted CRC cells (Figure 3). (pcMET was not examined in SW480 cells because this cell line does not express cMET.) Also, our observations indicated that levels of pSRC, a protein that interacts with FAK and plays 
A

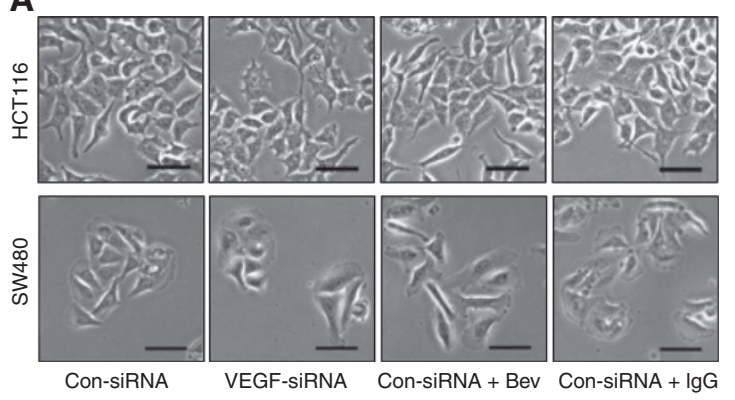

B

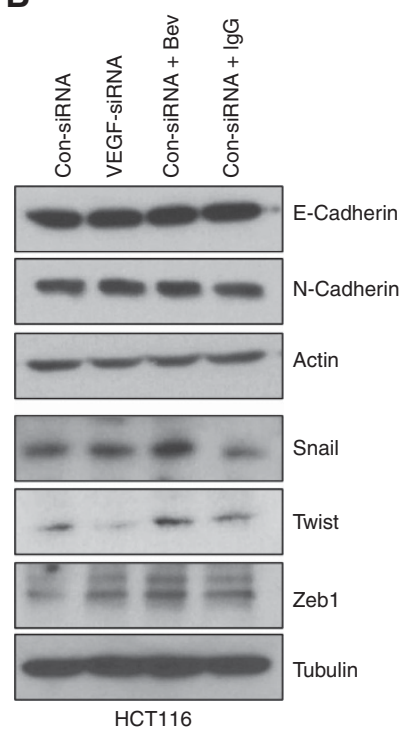

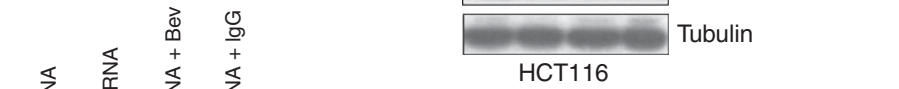

HCT116

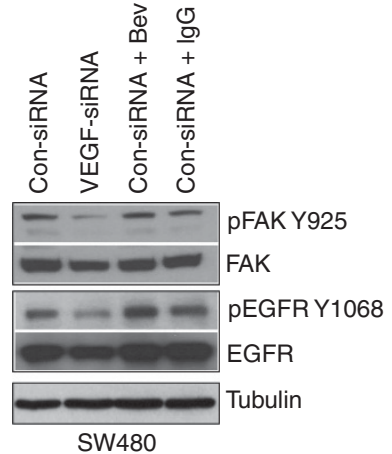

SW480

Figure 3. Markers of migration are altered in CRC cells depleted of VEGF. Cell lysates from CRC cells treated with Con-siRNA, VEGFsiRNA, Con-siRNA + Bev, or Con-siRNA + lgG were analysed for active FAK and its upstream activator pEGFR by western blot. Total FAK and EGFR levels are also shown. VEGF-siRNA-treated CRC cells had reduced $\mathrm{PFAK}$ and $\mathrm{pEGFR}$ levels compared with cells with normal VEGF levels. Representative $\alpha$-tubulin blots are shown as loading controls.

in control-siRNA-treated cells (Supplementary Figure 5), suggesting that pan-inhibition of tyrosine phosphatases is detrimental to cell migration.

Depletion of VEGFR1 induces strong inhibition of CRC cell migration. As our previous studies have suggested a role for a VEGF-VEGFR1 complex in intracrine signalling, we sought to determine whether depletion of VEGFR1 would inhibit CRC cell migration, as VEGF depletion did. Indeed, depleting VEGFR1 with siRNA in HCT116 and SW480 cells led to a strong inhibition of cell migration (Figure 4A), the degree of which was similar to the migration inhibition resulting from VEGF depletion (Figures 4A and 1B). To determine if the kinase activity of VEGFR1 was necessary for inhibition of CRC cell migration, CRC cells were also treated with two different inhibitors of VEGFR1, axitinib and pazopanib, at doses that inhibit VEGFR1 kinase phosphorylation (Supplementary Figure 6). These treatments failed to alter CRC cell migration, indicating an intracellular and kinase-independent function of VEGFR1 (Figure 4B). Further examination also revealed that VEGFR1 depletion (Figure 4C, left) led to insignificant changes in the EMT markers E-cadherin, N-cadherin, Twist, and Snail (Figure 4C, middle). However, VEGFR1 depletion strongly reduced the activity of the motility-involved proteins pFAK and pEGFR (Figure 4C, right), a finding similar to that in VEGF-depleted cells (Figure 3). We also noted decreased pcMET levels in the CRC cell lines that express cMET (data not shown). As the CRC cell lines examined have very low levels of or no VEGFR2 and VEGFR3 (Fan et al, 2005; Samuel et al, 2011), we did not study the role of those subtypes in CRC cell migration.

(Supplementary Figure 3), where VEGF depletion led to decreases in levels of pFAK and pcMET.

These findings corroborate with the effects of VEGF depletion in CRC cells as described before (Bhattacharya et al, 2016), that show activation of a yet unknown tyrosine phosphatase on VEGF depletion. This activation could explain the reduction of active cMET, FAK, and SRC levels in CRC cells. To establish this phosphatase mediated RTK inactivation as a molecular mechanism for mediating cell migration, we examined whether inhibiting tyrosine phosphatases can rescue the effects of VEGF depletion on cell migration in vitro. However, treating cells with low concentrations of $\mathrm{Na}_{3} \mathrm{VO}_{4}$, which specifically inhibits tyrosine phosphatases, not only failed to rescue the effects of VEGF depletion but also almost completely inhibited cell migration, even

Overexpression of cMET partially rescues inhibition of migration resulting from VEGF depletion. As our results indicated that VEGF depletion reduced the activity of pFAK and its upstream effectors, for example, pcMET, we sought to examine whether overexpression of cMET or FAK could rescue the effects of VEGF depletion. To validate that cMET is important in CRC cell migration, cMET in HCT116 cells was depleted using siRNA. cMET depletion led to a significant reduction in HCT116 cell migration (Supplementary Figure 7A). CRC cells transfected with control-siRNA or VEGF-siRNA were re-transfected with empty vector or a plasmid capable of expressing FLAG-cMET and were assayed for cell migration. As expected, VEGF-depleted cells had 
A
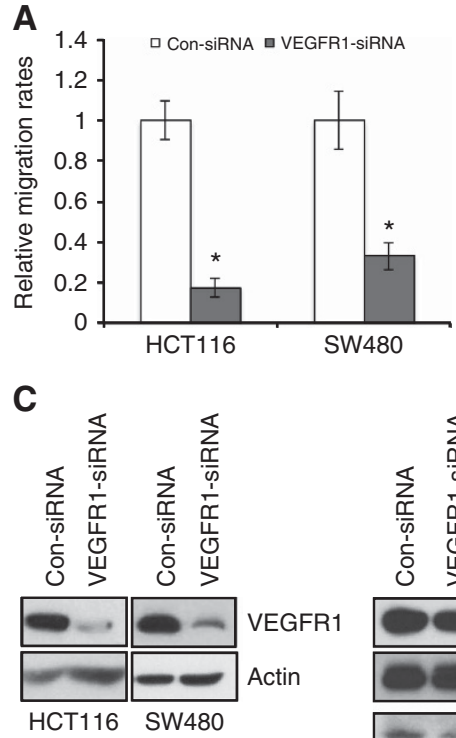

B

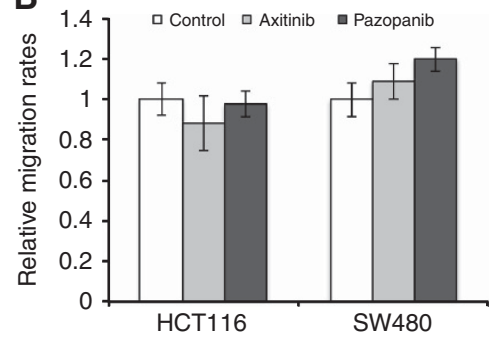

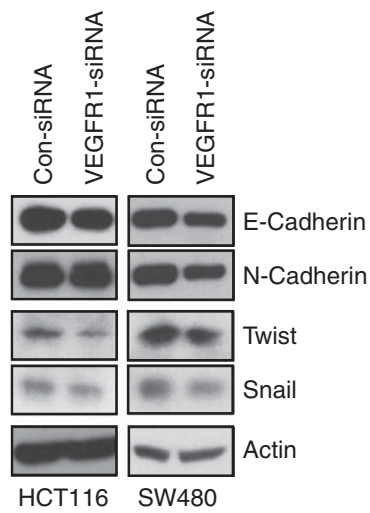

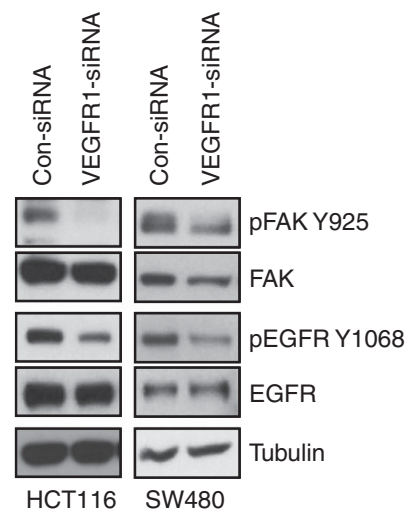

Figure 4. Depletion of VEGFR1 inhibits CRC cell migration and motility markers. (A) HCT116 and SW480 cells were transfected with siRNAs targeting human VEGF or VEGFR1 and assayed for migration using transwell migration chambers. A significant decrease in migration was observed in CRC cells depleted of VEGF (using VEGF-siRNA) or VEGFR1 (using VEGFR1-siRNA) compared with control cells (treated with ConsiRNA). The plots show relative migration rates derived from multiple experiments. ${ }^{\star} P<0.005$. (B) CRC cells were treated with or without the VEGFR1 inhibitors axitinib or pazopanib for $6 \mathrm{~h}$ and assayed for changes in cell migration using transwell migration chambers. The plots show relative migration rates as determined from multiple experiments. (C) CRC cells were transfected with Con-siRNA or VEGFR1-siRNA and assayed for alterations in levels of VEGFR1 (left panel), EMT markers (middle panel), and motility markers (right panel). VEGFR1-siRNAs reduced VEGFR1 and pFAK/pEGFR levels in CRC cells but did not alter levels of EMT markers. Single actin or $\alpha$-tubulin blots are shown as representative loading controls for simplicity.

significantly lower migration rates than control cells (Supplementary Figure 7B). Overexpression of FLAG-cMET in VEGF-depleted cells, however, significantly increased migration rates compared with the absence of FLAG-cMET overexpression and partially rescued the migratory defects arising from VEGF inhibition (Supplementary Figure 7B).

We attempted similar experiments with FAK. Treating CRC cells with the FAK inhibitor defactinib $(1 \mu \mathrm{M})$ resulted in a strong reduction of CRC cell migration (Supplementary Figure 7C), validating the essential role of FAK in CRC cell migration. To clearly establish if VEGF depletion-mediated reduction of FAK phosphorylation affected CRC cell migration, we attempted to overexpress FAK in VEGF-depleted cells to increase the cellular phospho-FAK levels and rescue the loss of migration. However, overexpression of GFP-FAK ( $\mathrm{Gu}$ et al, 1999) via a plasmid in VEGF-depleted CRC cells failed to rescue the defects in migration due to VEGF depletion (Supplementary Figure 7D). Surprisingly, overexpression of GFP-FAK in control-siRNA-treated cells also led to severe reduction of cell migration (Supplementary Figure 7D), suggesting that overexpression of FAK is detrimental to CRC cell migration.

\section{DISCUSSION}

In these studies, we have demonstrated that inhibition of intracellular VEGF severely affects CRC cell migration and invasion. These effects were due to inhibition of intracrine VEGF signalling, not paracrine or autocrine VEGF signalling, as evidenced by the negligible effects of adding bevacizumab to extracellular media during migration assays. We have also presented evidence that depletion of VEGFR1 leads to disruption of cell migration similar to that observed with VEGF depletion, suggesting an intracellular VEGF-VEGFR1 complex influencing cell motility and migration. Also, inhibition of VEGFR1 kinase function by kinase inhibitors had no significant effect on CRC cell migration, indicating that VEGFR1 kinase activity was not essential in regulating cell migration. EMT and changes in EMT markers have been described as major players with strong effects on tumour cell migration; however, we found neither morphologic changes nor changes in expression of EMT markers upon depletion of VEGF or VEGFR1. Changes in migration rates were finally attributed to alterations in the activity of motility markers (pFAK, pcMET, and pEGFR).

Although extensive studies have been done regarding the paracrine and autocrine cell-surface VEGF-VEGFR interactions and their effects on endothelial cell migration, few studies have explored intracrine VEGF signalling. Studies in breast cancer (Lee et al, 2007b), myeloma (Vincent et al, 2005) and CRC cells (Samuel et al, 2011; Bhattacharya et al, 2016) indicate that intracrine VEGF signalling plays an important role in tumour cell survival. Our present study clearly demonstrates that VEGF intracrine signalling also plays an important role in CRC cell migration and invasion. Our studies with other tumour cell types (Gastric, ovarian and breast cancer cells: Supplementary Figure 1) indicate that cell migration may be regulated by intracrine VEGF signalling in a variety of tumour cell types. Together, our findings support a general role of intracellular VEGF in modulating migration in tumour cells of various origins with active intracrine VEGF signalling.

The role of VEGF and its receptors in endothelial cell migration has been extensively studied (Koch and Claesson-Welsh, 2012). 
However, the role of these factors in tumour cell migration has been evaluated in only a few tumour types (Wey et al, 2004; Fan et al, 2005; Doi et al, 2012; Lu et al, 2012; Sennino et al, 2012). The current literature suggests that, regardless of cell type (endothelial cells or tumour cells), VEGF and its receptors regulate cell migration by signalling events initiated at the cell surface by VEGF binding to its receptors. In endothelial cells, the effects are direct, with VEGF-VEGFR2 complexes initiating signalling cascades that lead to cell migration through activation of p38 MAPK or FAK (Rousseau et al, 1997). Inhibition of VEGF-VEGFR2 signalling by VEGF-targeting antibodies or kinase inhibitors led to impaired endothelial cell migration (Hu-Lowe et al, 2008; Puddu et al, 2016). In tumour cells, the mechanism is more complex. Studies in glioblastoma cells have indicated that VEGF-VEGFR2 complex formation negatively regulates tumour cell migration by inactivation of cMET at the cell surface (Lu et al, 2012). A mechanism involving the direct interaction of VEGFR2 and cMET and the recruitment of protein tyrosine phosphatase $1 \mathrm{~B}$ to inactivate cMET was described. In these cells, inhibition of VEGF-VEGFR2 interaction by VEGF antibodies resulted in reduced VEGFR2cMET association and finally increased pcMET levels and migration rates. Similarly, in mouse models, pancreatic neuroendocrine tumour metastasis was increased in the presence of antiVEGF therapy but was efficiently blocked with anti-cMET treatment (Sennino et al, 2012). These studies imply a negative role of VEGF-VEGFR2 complexes in tumour cell migration, contrary to their role observed in endothelial cells. However, studies in pancreatic cancer cells have indicated that VEGFVEGFR2 signalling promotes pancreatic cancer cell migration that is reduced by treatment with anti-angiogenic agents bevacizumab and sunitinib (Doi et al, 2012).

On the basis of our studies, we propose that in CRC cells that lack VEGFR2, VEGF-VEGFR1 can regulate cell migration through a completely different and novel mechanism than the presently described paracrine/autocrine signalling mechanisms. This signalling occurs through an intracrine VEGF-VEGFR1 axis that regulates the activity of RTKs and FAK by sequestering a yetunknown tyrosine phosphatase. Normal VEGF or VEGFR1 expression in CRC cells inhibits the activity of this phosphatase or phosphatases and helps maintain normal levels of active pRTKs (pEGFR and pcMET) and pFAK. Depleting VEGF or VEGFR1 leads to activation of the phosphatase or phosphatases that, in turn, decreases pRTKs or pFAK and inhibits cell migration. Interestingly, contrary to all other VEGF-mediated migration effects described in endothelial cells or tumour cells, inhibition of cellsurface VEGF-VEGFR interactions has no effects on the intracrine VEGF signalling, as reported in this study.

One important observation resulted from one of the negative experiments performed during this study. In our efforts to prove that the VEGF-mediated regulation of phosphatase was responsible for the decrease in pRTKs and pFAK and led to inhibition of CRC cell migration, we treated control-siRNA-transfected or VEGFsiRNA-transfected cells with minimal effective doses of tyrosine phosphatase inhibitor $\mathrm{Na}_{3} \mathrm{VO}_{4}$. Similar experiments have shown that the phospho-protein levels were successfully rescued and often enhanced in VEGF-depleted cells (Bhattacharya et al, 2016) and have validated the mechanism involving a VEGF-mediated phosphatase. However, all cells treated with $\mathrm{Na}_{3} \mathrm{VO}_{4}$ to inactivate tyrosine phosphatases and regardless of VEGF levels, failed to migrate in the transwell migration assays. This indicated that lack of dephosphorylation of key proteins may have led to failure in cell migration. These results indicate and validate the importance of having the right balance of phosphorylated proteins for different cellular processes, including cell migration.

It is widely believed that VEGF-targeted therapies primarily effect endothelial cells, but results from clinical trials have not been as successful in improving patient survival as expected (Jayson et al, 2016). Our previous studies have indicated that targeting intracellular VEGF in tumour cells makes tumour cells more sensitive to chemotherapy (Samuel et al, 2011; Bhattacharya et al, 2016) and thus may be a novel means of targeting tumour cells rather than the tumour vasculature. Also, a clinical study determining the efficacy of VEGF and KSP1 siRNAs showed no significant toxicity due to VEGF siRNAs in the patients, but one patient exhibited a complete response, a result that is rarely if ever observed with current U.S. Food and Drug Administrationapproved VEGF-targeted therapies when used as a single agent (Tabernero et al, 2013). Our present study indicates that targeting intracellular VEGF in tumour cells can reduce tumour cell migration and invasion. These results make a strong case for possible future use of VEGF siRNAs in combination with chemotherapy, instead of present anti-angiogenic and chemotherapy combinations, as neoadjuvant therapy for patients who have advanced disease.

\section{ACKNOWLEDGEMENTS}

This work was supported in part by the Gillson-Longenbaugh Foundation (LME and RB), the William C. Liedtke, Jr., Chair in Cancer Research (LME), National Institutes of Health grant R01CA157880 (LME), and National Institutes of Health Cancer Center Support Grant CA016672. We also would like to thank Dr Mien-Chie Hung for the FLAG-cMET plasmid, The Department of Scientific Publications at MD Anderson Cancer Center for editing the manuscript, and Ms. Rita Hernandez for support in preparing the manuscript.

\section{CONFLICT OF INTEREST}

Lee M Ellis serves as an ad hoc consultant for Genentech/Roche.

\section{REFERENCES}

Anderson JM, Fanning AS, Lapierre L, Van Itallie CM (1995) Zonula occludens (ZO)-1 and ZO-2: membrane-associated guanylate kinase homologues (MAGuKs) of the tight junction. Biochem Soc Trans 23(3): 470-475.

Bhattacharya R, Ye XC, Wang R, Ling X, McManus M, Fan F, Boulbes D, Ellis LM (2016) Intracrine VEGF signaling mediates the activity of prosurvival pathways in human colorectal cancer cells. Cancer Res 76(10): 3014-3024.

Cano A, Perez-Moreno MA, Rodrigo I, Locascio A, Blanco MJ, del Barrio MG, Portillo F, Nieto MA (2000) The transcription factor snail controls epithelial-mesenchymal transitions by repressing E-cadherin expression. Nat Cell Biol 2(2): 76-83.

Connolly DT, Heuvelman DM, Nelson R, Olander JV, Eppley BL, Delfino JJ, Siegel NR, Leimgruber RM, Feder J (1989) Tumor vascular permeability factor stimulates endothelial cell growth and angiogenesis. J Clin Invest 84(5): 1470-1478.

Costa R, Carneiro A, Rocha A, Pirraco A, Falcao M, Vasques L, Soares R (2009) Bevacizumab and ranibizumab on microvascular endothelial cells: a comparative study. J Cell Biochem 108(6): 1410-1417.

Deramaudt TB, Dujardin D, Hamadi A, Noulet F, Kolli K, De Mey J, Takeda K, Ronde P (2011) FAK phosphorylation at Tyr-925 regulates cross-talk between focal adhesion turnover and cell protrusion. Mol Biol Cell 22(7): 964-975.

Doi Y, Yashiro M, Yamada N, Amano R, Noda S, Hirakawa K (2012) VEGFA/VEGFR-2 signaling plays an important role for the motility of pancreas cancer cells. Ann Surg Oncol 19(8): 2733-2743.

Du Y, Yamaguchi H, Wei Y, Hsu JL, Wang HL, Hsu YH, Lin WC, Yu WH, Leonard PG, Lee GRt, Chen MK, Nakai K, Hsu MC, Chen CT, Sun Y, Wu Y, Chang WC, Huang WC, Liu CL, Chang YC, Chen CH, Park M, Jones P, Hortobagyi GN, Hung MC (2016) Blocking c-Met-mediated 
PARP1 phosphorylation enhances anti-tumor effects of PARP inhibitors. Nat Med 22(2): 194-201.

Eger A, Aigner K, Sonderegger S, Dampier B, Oehler S, Schreiber M, Berx G, Cano A, Beug H, Foisner R (2005) DeltaEF1 is a transcriptional repressor of E-cadherin and regulates epithelial plasticity in breast cancer cells. Oncogene 24(14): 2375-2385.

Fan F, Wey JS, McCarty MF, Belcheva A, Liu W, Bauer TW, Somcio RJ, Wu Y, Hooper A, Hicklin DJ, Ellis LM (2005) Expression and function of vascular endothelial growth factor receptor-1 on human colorectal cancer cells. Oncogene 24(16): 2647-2653.

Ferrara N, Carver-Moore K, Chen H, Dowd M, Lu L, O'Shea KS, Powell-Braxton L, Hillan KJ, Moore MW (1996) Heterozygous embryonic lethality induced by targeted inactivation of the VEGF gene. Nature 380(6573): 439-442.

Ferrara N, Gerber HP, LeCouter J (2003) The biology of VEGF and its receptors. Nat Med 9(6): 669-676.

Gerber HP, Dixit V, Ferrara N (1998a) Vascular endothelial growth factor induces expression of the antiapoptotic proteins $\mathrm{Bcl}-2$ and $\mathrm{A} 1$ in vascular endothelial cells. J Biol Chem 273(21): 13313-13316.

Gerber HP, Hillan KJ, Ryan AM, Kowalski J, Keller GA, Rangell L, Wright BD, Radtke F, Aguet M, Ferrara N (1999) VEGF is required for growth and survival in neonatal mice. Development 126(6): 1149-1159.

Gerber HP, McMurtrey A, Kowalski J, Yan M, Keyt BA, Dixit V, Ferrara N (1998b) Vascular endothelial growth factor regulates endothelial cell survival through the phosphatidylinositol 3'-kinase/Akt signal transduction pathway. Requirement for Flk-1/KDR activation. J Biol Chem 273(46): 30336-30343.

Gu J, Tamura M, Pankov R, Danen EH, Takino T, Matsumoto K, Yamada KM (1999) Shc and FAK differentially regulate cell motility and directionality modulated by PTEN. J Cell Biol 146(2): 389-403.

Hu-Lowe DD, Zou HY, Grazzini ML, Hallin ME, Wickman GR, Amundson K, Chen JH, Rewolinski DA, Yamazaki S, Wu EY, McTigue MA, Murray BW, Kania RS, O'Connor P, Shalinsky DR, Bender SL (2008) Nonclinical antiangiogenesis and antitumor activities of axitinib (AG-013736), an oral, potent, and selective inhibitor of vascular endothelial growth factor receptor tyrosine kinases 1, 2, 3. Clin Cancer Res 14(22): 7272-7283.

Jayson GC, Kerbel R, Ellis LM, Harris AL (2016) Antiangiogenic therapy in oncology: current status and future directions. Lancet 388(10043): 518-529.

Kalluri R, Weinberg RA (2009) The basics of epithelial-mesenchymal transition. J Clin Invest 119(6): 1420-1428.

Koch S, Claesson-Welsh L (2012) Signal transduction by vascular endothelial growth factor receptors. Cold Spring Harb Perspect Med 2(7): a006502.

Lee S, Chen TT, Barber CL, Jordan MC, Murdock J, Desai S, Ferrara N, Nagy A, Roos KP, Iruela-Arispe ML (2007a) Autocrine VEGF signaling is required for vascular homeostasis. Cell 130(4): 691-703.

Lee TH, Seng S, Sekine M, Hinton C, Fu Y, Avraham HK, Avraham S (2007b) Vascular endothelial growth factor mediates intracrine survival in human breast carcinoma cells through internally expressed VEGFR1/FLT1. PLoS Med 4(6): e186.

Leung DW, Cachianes G, Kuang WJ, Goeddel DV, Ferrara N (1989) Vascular endothelial growth factor is a secreted angiogenic mitogen. Science 246(4935): 1306-1309.

Lu KV, Chang JP, Parachoniak CA, Pandika MM, Aghi MK, Meyronet D, Isachenko N, Fouse SD, Phillips JJ, Cheresh DA, Park M, Bergers G (2012) VEGF inhibits tumor cell invasion and mesenchymal transition through a MET/VEGFR2 complex. Cancer Cell 22(1): 21-35.

Mitra SK, Hanson DA, Schlaepfer DD (2005) Focal adhesion kinase: in command and control of cell motility. Nat Rev Mol Cell Biol 6(1): 56-68.

Mitra SK, Mikolon D, Molina JE, Hsia DA, Hanson DA, Chi A, Lim ST, Bernard-Trifilo JA, Ilic D, Stupack DG, Cheresh DA, Schlaepfer DD (2006) Intrinsic FAK activity and Y925 phosphorylation facilitate an angiogenic switch in tumors. Oncogene 25(44): 5969-5984.

Mitra SK, Schlaepfer DD (2006) Integrin-regulated FAK-Src signaling in normal and cancer cells. Curr Opin Cell Biol 18(5): 516-523.
Muller T, Bain G, Wang X, Papkoff J (2002) Regulation of epithelial cell migration and tumor formation by beta-catenin signaling. Exp Cell Res 280(1): 119-133.

Plouet J, Schilling J, Gospodarowicz D (1989) Isolation and characterization of a newly identified endothelial cell mitogen produced by AtT-20 cells. EMBO J 8(12): 3801-3806.

Podar K, Tonon G, Sattler M, Tai YT, Legouill S, Yasui H, Ishitsuka K, Kumar S, Kumar R, Pandite LN, Hideshima T, Chauhan D, Anderson KC (2006) The small-molecule VEGF receptor inhibitor pazopanib (GW786034B) targets both tumor and endothelial cells in multiple myeloma. Proc Natl Acad Sci USA 103(51): 19478-19483.

Puddu A, Sanguineti R, Traverso CE, Viviani GL, Nicolo M (2016) Response to anti-VEGF-A treatment of endothelial cells in vitro. Exp Eye Res 146: 128-136.

Rousseau S, Houle F, Landry J, Huot J (1997) p38 MAP kinase activation by vascular endothelial growth factor mediates actin reorganization and cell migration in human endothelial cells. Oncogene 15(18): 2169-2177.

Samuel S, Fan F, Dang LH, Xia L, Gaur P, Ellis LM (2011) Intracrine vascular endothelial growth factor signaling in survival and chemoresistance of human colorectal cancer cells. Oncogene 30(10): 1205-1212.

Scheel C, Eaton EN, Li SH, Chaffer CL, Reinhardt F, Kah KJ, Bell G, Guo W, Rubin J, Richardson AL, Weinberg RA (2011) Paracrine and autocrine signals induce and maintain mesenchymal and stem cell states in the breast. Cell 145(6): 926-940.

Scheel C, Weinberg RA (2012) Cancer stem cells and epithelial-mesenchymal transition: concepts and molecular links. Semin Cancer Biol 22(5-6): 396-403.

Sennino B, Ishiguro-Oonuma T, Wei Y, Naylor RM, Williamson CW, Bhagwandin V, Tabruyn SP, You WK, Chapman HA, Christensen JG, Aftab DT, McDonald DM (2012) Suppression of tumor invasion and metastasis by concurrent inhibition of c-Met and VEGF signaling in pancreatic neuroendocrine tumors. Cancer Discov 2(3): 270-287.

Tabernero J, Shapiro GI, LoRusso PM, Cervantes A, Schwartz GK, Weiss GJ, Paz-Ares L, Cho DC, Infante JR, Alsina M, Gounder MM, Falzone R, Harrop J, White AC, Toudjarska I, Bumcrot D, Meyers RE, Hinkle G, Svrzikapa N, Hutabarat RM, Clausen VA, Cehelsky J, Nochur SV, Gamba-Vitalo C, Vaishnaw AK, Sah DW, Gollob JA, Burris 3rd HA (2013) First-in-humans trial of an RNA interference therapeutic targeting VEGF and KSP in cancer patients with liver involvement. Cancer Discov 3(4): 406-417.

Thiery JP, Sleeman JP (2006) Complex networks orchestrate epithelialmesenchymal transitions. Nat Rev Mol Cell Biol 7(2): 131-142.

Vincent L, Jin DK, Karajannis MA, Shido K, Hooper AT, Rashbaum WK, Pytowski B, Wu Y, Hicklin DJ, Zhu Z, Bohlen P, Niesvizky R, Rafii S (2005) Fetal stromal-dependent paracrine and intracrine vascular endothelial growth factor-a/vascular endothelial growth factor receptor-1 signaling promotes proliferation and motility of human primary myeloma cells. Cancer Res 65(8): 3185-3192.

Wey JS, Stoeltzing O, Ellis LM (2004) Vascular endothelial growth factor receptors: expression and function in solid tumors. Clin Adv Hematol Oncol 2(1): 37-45.

Yang J, Mani SA, Donaher JL, Ramaswamy S, Itzykson RA, Come C, Savagner P, Gitelman I, Richardson A, Weinberg RA (2004) Twist, a master regulator of morphogenesis, plays an essential role in tumor metastasis. Cell 117(7): 927-939.

Yang J, Weinberg RA (2008) Epithelial-mesenchymal transition: at the crossroads of development and tumor metastasis. Dev Cell 14(6): 818-829.

Zhao X, Guan JL (2011) Focal adhesion kinase and its signaling pathways in cell migration and angiogenesis. Adv Drug Deliv Rev 63(8): 610-615.

This work is published under the standard license to publish agreement. After 12 months the work will become freely available and the license terms will switch to a Creative Commons AttributionNonCommercial-Share Alike 4.0 Unported License.

Supplementary Information accompanies this paper on British Journal of Cancer website (http://www.nature.com/bjc) 